\section{Efeito da fortificação de alimentos com ferro sobre anemia em crianças: um estudo de revisão}

\author{
Effect of food fortification with iron on \\ childhood anemia: a review study
}

Faculdade de Nutrição,
Universidade Federal de
Pelotas, Pelotas, Brasil.
2 Faculdade de Medicina,
Universidade Federal de
Pelotas, Pelotas, Brasil.
Correspondência
M. C. F. Assunção
Programa de Pós-graduação
em Epidemiologia, Faculdade
de Medicina, Universidade
Federal de Pelotas.
Av. Duque de Caxias 250,
Pelotas, RS
96030-00o, Brasil.
mcassuncao@epidemio-ufpel.
org.br

\section{Abstract}

A systematic review was conducted to identify studies assessing the effect of food fortification with iron on childhood anemia. The MEDLINE, LILACS, and PubMed databases and WHO and PAHO sites were searched with no time limitation, including articles published in Portuguese, English, or Spanish, using the following key words and their combination: food fortification, iron, effectiveness, efficacy, anemia, flour, staple foods, interventions, and children. Of 21 studies reviewed, only one failed to report a positive, favorable effect of iron fortification, indicating the possibility of publication bias. The studies showed important methodological limitations. The two studies with the best methodological scores showed opposite results, highlighting the need for larger trials with better planning to explore this hypothesis.

Fortified Food; Iron; Anemia; Child Welfare
Maria Cecília F. Assunção 1,2

Iná S. Santos 2

\section{Introdução}

A deficiência de ferro, incluindo a sua forma mais severa, a anemia, é a carência nutricional mais comum no mundo, sendo mais prevalente em grupos populacionais que têm altos requerimentos de ferro. Crianças e mulheres em idade reprodutiva são os grupos mais vulneráveis à ocorrência dessa condição ${ }^{1}$.

A Organização Mundial da Saúde (OMS) estima que o número de pessoas anêmicas em todo o mundo aproxime-se de dois bilhões e que a maioria dos casos de anemia seja causada por deficiência de ferro ${ }^{2}$. No Brasil, estudos de base populacional mostram prevalências de anemia superiores a $30 \% 3,4,5,6,7,8,9,10,11,12$.

Dentre as estratégias utilizadas para prevenção da anemia por deficiência de ferro, encontrase a fortificação de alimentos, apontada como a melhor abordagem para aumentar a ingestão desse mineral 13. Segundo a OMS, são reconhecidos quatro tipos de fortificação. A "fortificação em massa ou universal" que consiste na adição de micronutrientes aos alimentos consumidos pela grande maioria da população, sendo regulada pelos governos. É indicada em países onde diferentes grupos populacionais apresentam risco elevado para desenvolvimento de anemia. A fortificação em "mercado aberto", por iniciativa da indústria de alimentos em fortificar seus produtos, com o objetivo de aumentar seus lucros. A "fortificação direcionada”, que consiste na forti- 
ficação de alimentos consumidos por grupos de alto-risco para anemia e a "fortificação comunitária ou domiciliar", uma abordagem recente que está sendo explorada em países em desenvolvimento, em que são adicionados suplementos às refeições das crianças 14 .

A fortificação universal com ferro em alimentos comumente consumidos tem sido utilizada em muitos países por representar uma solução prática e de baixo custo 15 para combater a carência do mineral. Farinhas de cereais são os alimentos mais comumente fortificados, mas outros, tais como massas, arroz e vários tipos de molhos 16 também são utilizados. A exemplo de outros países 17, o Brasil, desde junho de 2004, tornou obrigatória a fortificação das farinhas de trigo e milho com ferro e ácido fólico 18 .

O presente artigo é uma revisão de publicações que descrevem a eficácia e/ou a efetividade de intervenções com fortificação de alimentos, endereçadas a crianças e adolescentes, cujo desfecho tenha sido anemia ou indicadores dos estoques de ferro no organismo.

\section{Metodologia}

A revisão bibliográfica foi realizada nas bases de dados MEDLINE, LILACS e nos sites da OMS e Organização Pan-Americana da Saúde (OPAS), sem limite de tempo, incluindo estudos publicados em português, inglês ou espanhol, utilizando os seguintes unitermos e suas combinações: food fortification, iron, effectiveness, efficacy, anemia, flour, staple foods, interventions e children.

Após a identificação e leitura de 32 resumos, foram selecionados 15 artigos. Destes, foram checadas as referências e identificados mais seis estudos inicialmente não encontrados nas bases de dados pesquisadas. Os artigos revisados foram analisados e pontuados conforme os critérios de Downs \& Black 19.

\section{Resultados}

Foram incluídos nesta revisão 21 estudos que avaliaram a eficácia ou a efetividade de medidas de fortificação de alimentos com ferro. Dos 11 estudos internacionais 20,21,22,23,24,25,26,27,28,29,30 incluídos nesta revisão, nove foram randomizados 20,21,22,23,25,27,28,29,30, um do tipo quase-experimental e outro uma avaliação transversal. Dos dez nacionais $31,32,33,34,35,36,37,38,39,40$, nove foram do tipo antes e depois e apenas um foi randomizado 39. As Tabelas 1 e 2 mostram os principais aspectos dos estudos selecionados.

\section{Estudos internacionais}

No Chile, Hertrampf et al. 20 avaliaram o efeito da ingestão de leite em pó fortificado com ferro na forma de sulfato ferroso (15mg de ferro/ $100 \mathrm{~g}$ de pó) em um estudo prospectivo com 229 lactentes acompanhados em um ambulatório público dos 3 aos 12 meses de vida. Estes foram alocados ao acaso em dois grupos: 114 receberam leite em pó fortificado e 115 receberam leite sem adição de ferro. Semanalmente, eram realizadas visitas domiciliares com aplicação de recordatórios alimentares de 24 horas com o objetivo de medir a aceitabilidade das fórmulas. Os grupos que receberam leite fortificado e não-fortificado ingeriram $94 \%$ e $95 \%$ da quantidade de leite indicada durante todo o estudo, respectivamente. Amostras de sangue venoso foram obtidas aos 9 e 12 meses de idade. Trinta e nove crianças foram perdidas no acompanhamento, 28 no grupo intervenção e 11 no grupo controle. O grupo que recebeu a fortificação apresentou valores significativamente maiores de todos os indicadores de status de ferro nas duas avaliações. Ao final de 12 meses de estudo, nenhuma criança anêmica foi encontrada no grupo que recebeu fortificação, enquanto que $34 \%$ das crianças do grupo que não recebeu fortificação encontravam-se anêmicas. A comparabilidade dos dois grupos quanto ao status dos indicadores hematimétricos no início do estudo não foi relatada pelos autores.

Hertrampf et al. ${ }^{21}$, também no Chile, testaram o efeito de uma farinha de arroz fortificada com ferro $(14 \mathrm{mg} / 100 \mathrm{~g})$ proveniente de hemoglobina bovina. Eram elegíveis para o estudo um total de 239 crianças de baixo nível sócio-econômico, que estavam sendo amamentadas aos três meses de vida e eram atendidas em um ambulatório público da capital do país. Houve perda de $21 \%$ da amostra inicial, sendo incluídas 188 crianças. Dessas, 92 foram alocadas aleatoriamente para receberem o cereal fortificado na quantidade de $20 \mathrm{~g} /$ dia até os quatro meses de idade e de $40 \mathrm{~g} /$ dia a partir dos seis meses. As 96 crianças que faziam parte do grupo controle receberam recomendações sobre alimentação de acordo com a prática usual do país. Visitas domiciliares foram realizadas semanalmente para checar a adesão ao consumo de cereais, sendo observado que das crianças que receberam o cereal fortificado, $34 \%$ consumiram menos de 20g/dia e $66 \%$ no mínimo $20 \mathrm{~g} /$ dia. Do grupo de crianças com mais de seis meses, 31 crianças $(34 \%)$ consumiram o cereal em quantidades superiores a $30 \mathrm{~g} / \mathrm{dia}$, o que os autores consideraram adequado. Amostras de sangue venoso obtidas aos 9 e 12 meses de idade mostraram valores significativamente maiores da taxa de saturação da transferrina e ferritina sérica 
Resumo dos estudos internacionais de avaliação do impacto da fortificação de alimentos com ferro sobre a ocorrência de anemia.

\begin{tabular}{|c|c|c|c|c|c|c|c|}
\hline Referência & $\begin{array}{c}\text { Ano de } \\
\text { publicação }\end{array}$ & $\begin{array}{l}\text { Local de } \\
\text { estudo }\end{array}$ & Amostra & $\begin{array}{l}\text { Tipo de } \\
\text { estudo }\end{array}$ & $\begin{array}{l}\text { Duração } \\
\text { (meses) }\end{array}$ & Resultado & $\begin{array}{l}\text { Alimento } \\
\text { fortificado }\end{array}$ \\
\hline $\begin{array}{l}\text { Hertrampf } \\
\text { et al. } 20\end{array}$ & 1990 & Chile & $\begin{array}{l}229 \text { crianças; } 3 \\
\text { meses de idade; } \\
1 \text { ambulatório } \\
\text { público }\end{array}$ & Randomizado & 9 & $\begin{array}{l}\text { Aos } 9 \text { e } 12 \text { meses de idade o grupo que } \\
\text { recebeu leite fortificado apresentou valores } \\
\text { significativamente maiores de hemoglobina, } \\
\text { volume corpuscular médio, ferro sérico, e } \\
\text { capacidade total de saturação da transferrina e } \\
\text { ferritina sérica do que o controle }\end{array}$ & Leite em pó \\
\hline $\begin{array}{l}\text { Hertrampf } \\
\text { et al. } 21\end{array}$ & 1990 & Chile & $\begin{array}{l}239 \text { crianças; } \\
3 \text { meses; } 1 \\
\text { ambulatório; } \\
\text { baixo nível } \\
\text { socio-econômico; } \\
\text { recebendo leite } \\
\text { materno }\end{array}$ & Randomizado & 9 & $\begin{array}{l}\text { Ao final de } 12 \text { meses, anemia estava presente em } \\
17 \% \text { e } 10 \% \text { das crianças dos grupos controle e } \\
\text { intervenção, respectivamente, e em } 6 \% \text { das } \\
\text { crianças que ingeriram acima de } 30 \mathrm{~g} / \text { dia do } \\
\text { cereal }\end{array}$ & $\begin{array}{c}\text { Farinha de } \\
\text { arroz }\end{array}$ \\
\hline $\begin{array}{l}\text { Olivares } \\
\text { et al. } 22\end{array}$ & 1990 & Chile & $\begin{array}{l}427 \text { crianças; } \\
6 \text { a } 12 \text { anos; } 2 \\
\text { escolas de áreas } \\
\text { de baixa renda }\end{array}$ & Randomizado & $\begin{array}{c}15 \\
\text { (2 perío- } \\
\text { dos esco- } \\
\text { lares) }\end{array}$ & $\begin{array}{l}\text { Ao final do estudo ( } 15 \text { meses), a concentração de } \\
\text { hemoglobina foi significativamente mais alta no } \\
\text { grupo fortificado }\end{array}$ & $\begin{array}{l}\text { Biscoitos de } \\
\text { farinha de } \\
\text { trigo }\end{array}$ \\
\hline $\begin{array}{l}\text { Walter } \\
\text { et al. } 23\end{array}$ & 1993 & Chile & $\begin{array}{l}515 \text { crianças; } 4 \\
\text { meses, divididos } \\
\text { em } 2 \text { grupos, } \\
\text { conforme } \\
\text { status de } \\
\text { amamentação; } \\
1 \text { ambulatório } \\
\text { público }\end{array}$ & Randomizado & 11 & $\begin{array}{l}\text { O grupo de crianças não amamentadas que } \\
\text { recebeu cereal ou fórmula fortificada apresentou } \\
\text { valores significativamente mais altos de } \\
\text { hemoglobina nos três acompanhamentos, } \\
\text { quando comparadas ao grupo que não recebeu } \\
\text { fortificação. Não houve diferença significativa na } \\
\text { concentração de hemoglobina entre o grupo que } \\
\text { recebeu cereal e o grupo que recebeu fórmula } \\
\text { fortificada. Entre as crianças amamentadas, a } \\
\text { concentração de hemoglobina foi mais alta aos } 12 \\
\text { e } 15 \text { meses no grupo que recebeu leite materno } \\
\text { mais cereal fortificado }\end{array}$ & $\begin{array}{c}\text { Cereal à } \\
\text { base de arroz } \\
\text { e/ou fórmula } \\
\text { infantil }\end{array}$ \\
\hline $\begin{array}{l}\text { Walter } \\
\text { et al. } 24\end{array}$ & 1993 & Chile & $\begin{array}{l}933 \text { adoles- } \\
\text { centes; } 10 \text { a } 16 \\
\text { anos; } 8 \text { escolas } \\
\text { de } 2 \text { províncias }\end{array}$ & $\begin{array}{c}\text { Quase- } \\
\text { experimento }\end{array}$ & 36 & $\begin{array}{l}\text { Média de hemoglobina e ferritina sérica no grupo } \\
\text { que recebeu intervenção foi maior do que no } \\
\text { grupo controle }\end{array}$ & Biscoitos \\
\hline Viteri et al. 25 & 1995 & Guatemala & $\begin{array}{l}346 \text { crianças; } \\
1 \text { a } 8 \text { anos; } 4 \\
\text { comunidades }\end{array}$ & Randomizado & 32 & $\begin{array}{l}\text { Nas três comunidades que sofreram intervenção } \\
\text { houve manutenção ou aumento significativo dos } \\
\text { indicadores do status de ferro. Na comunidade } \\
\text { controle não houve aumento significativo entre } \\
\text { a análise basal e ao final do estudo, exceto na } \\
\text { capacidade de saturação da transferrina }\end{array}$ & Açúcar \\
\hline $\begin{array}{l}\text { Layrisse } \\
\text { et al. } 26\end{array}$ & 1996 & Venezuela & $\begin{array}{l}317 \text { adoles- } \\
\text { centes escolares; } \\
7,11 \text { e } 15 \text { anos }\end{array}$ & Transversal & - & $\begin{array}{l}\text { Concentração de ferritina sérica e prevalência } \\
\text { de anemia foram reduzidas após dois anos de } \\
\text { consumo de farinhas fortificadas }\end{array}$ & $\begin{array}{l}\text { Farinhas de } \\
\text { milho e de } \\
\text { trigo }\end{array}$ \\
\hline
\end{tabular}

(continua) 


\begin{tabular}{|c|c|c|c|c|c|c|c|}
\hline Referência & $\begin{array}{c}\text { Ano de } \\
\text { publicação }\end{array}$ & $\begin{array}{l}\text { Local de } \\
\text { estudo }\end{array}$ & Amostra & $\begin{array}{l}\text { Tipo de } \\
\text { estudo }\end{array}$ & $\begin{array}{l}\text { Duração } \\
\text { (meses) }\end{array}$ & Resultado & $\begin{array}{l}\text { Alimento } \\
\text { fortificado }\end{array}$ \\
\hline $\begin{array}{l}\text { Walter } \\
\text { et al. } 27\end{array}$ & 1998 & Chile & $\begin{array}{l}835 \text { lactentes; } \\
6 \text { meses; sem } \\
\text { anemia; nascidos } \\
\text { a termo; } \\
\text { exclusivamente } \\
\text { ou parcialmente } \\
\text { amamentados }\end{array}$ & Randomizado & 6 & $\begin{array}{l}\text { A análise mostrou que as duas fórmulas foram } \\
\text { efetivas na prevenção da anemia. Não houve } \\
\text { diferença na prevalência após seis meses } \\
\text { de consumo de fórmulas com alta ou baixa } \\
\text { concentração de ferro }\end{array}$ & $\begin{array}{c}\text { Fórmulas } \\
\text { lácteas } \\
\text { enriquecidas } \\
\text { com alta } \\
\text { e baixa } \\
\text { concentração } \\
\text { de ferro }\end{array}$ \\
\hline Sari et al. 28 & 2001 & Indonésia & $\begin{array}{l}117 \text { crianças; } 4 \\
\text { a } 6 \text { anos; nível } \\
\text { sócio-econômico } \\
\text { baixo ou médio; } \\
4 \text { escolas infantis }\end{array}$ & Randomizado & 3 & $\begin{array}{l}\text { Aumento médio da concentração de hemoglobina } \\
\text { e ferritina sérica foi maior no grupo intervenção. } \\
\text { A prevalência de anemia passou de } 43,3 \% \text { para } \\
26,7 \% \text { no grupo controle, e de } 50,9 \% \text { para } 8,8 \% \\
\text { no grupo intervenção }\end{array}$ & Balas \\
\hline $\begin{array}{l}\text { Nestel } \\
\text { et al. } 29\end{array}$ & 2004 & Sri Lanka & $\begin{array}{l}1.103 \text { pré- } \\
\text { escolares; } 9-71 \\
\text { meses; } 1.004 \\
\text { escolares, } 6 \text { a } 11 \\
\text { anos }\end{array}$ & Randomizado & 24 & $\begin{array}{l}\text { Não houve diferença nos níveis de hemoglobina } \\
\text { após } 12 \text { e } 24 \text { meses de consumo de farinhas } \\
\text { fortificadas }\end{array}$ & $\begin{array}{l}\text { Farinha de } \\
\text { trigo }\end{array}$ \\
\hline $\begin{array}{l}\text { van } \\
\text { Stuijvenberg } \\
\text { et al. } 30\end{array}$ & 2006 & África do Sul & $\begin{array}{l}160 \text { crianças; } 1 \\
\text { escola }\end{array}$ & Randomizado & 7,5 & $\begin{array}{l}\text { Aumento significativo nos níveis de hemoglobina, } \\
\text { saturação de transferrina, ferritina e ferro sérico foi } \\
\text { observado no grupo que recebeu pão fortificado } \\
\text { com biglicinato ferroso, quando comparado ao } \\
\text { grupo que recebeu pão fortificado com ferro } \\
\text { eletrolítico e ao grupo controle }\end{array}$ & Pão preto \\
\hline
\end{tabular}

no grupo intervenção. Ao final de 12 meses, anemia estava presente em $17 \%$ e $10 \%$ das crianças do grupo controle e intervenção, respectivamente. Apenas $6 \%$ das crianças que ingeriram acima de $30 \mathrm{~g} /$ dia do cereal estavam anêmicas ao final da intervenção.

Olivares et al. 22 estudaram o efeito sobre o status de ferro da utilização de biscoitos produzidos com farinha de trigo fortificada com $6 \%$ de concentrado de hemoglobina bovina. Inicialmente, 427 escolares de seis a doze anos de duas escolas situadas em comunidades de baixa renda em Santiago, no Chile, foram incluídos no estudo. As crianças de uma escola (intervenção) receberam três unidades diárias de biscoito fortificado (30g), junto com um copo de leite. Na outra escola (controle) as crianças receberam a mesma quantidade diária de leite e biscoito, porém sem adição de ferro. Essa distribuição era supervisionada pelas professoras. Os alunos deixavam no refeitório os biscoitos não consumidos. Uma enfermeira treinada observava a aceitação dos biscoitos visitando semanalmente as escolas. A duração do estudo foi de 15 meses, com interrup- ção de três meses no período de férias escolares. Sessenta e três crianças (15\%) foram perdidas no acompanhamento, sendo 34 do grupo intervenção e 29 do grupo controle. Sangue venoso para determinação dos indicadores do status de ferro foi obtido no início, aos sete e aos 15 meses do estudo. A aceitabilidade dos biscoitos foi considerada excelente nos dois grupos. A avaliação inicial mostrou que os grupos eram similares em termos de concentração de hemoglobina $(13,83 \mathrm{~g} / \mathrm{dL}$ e 13,17g/dL nos grupos intervenção e controle, respectivamente) e ferritina $(27 \mathrm{mcg} / \mathrm{L}$ nos dois grupos). O índice de saturação de transferrina era mais alto no grupo não-fortificado $(24,6 \%$ e $26,8 \%$, respectivamente, nos grupos intervenção e controle). Aos sete meses, somente a concentração de ferritina sérica foi maior no grupo fortificado (31mcg/L vs. 26mcg/L; p < 0,01). Ao final do estudo (15 meses), a concentração de hemoglobina foi mais alta no grupo fortificado $(13,65 \pm 0,69 \mathrm{~g} / \mathrm{dL} v s .13,48 \pm 0,82 \mathrm{~g} / \mathrm{dL} ; \mathrm{p}<0,01)$.

Ainda no Chile, Walter et al. 23 verificaram a efetividade do uso de um cereal infantil à base de arroz fortificado com ferro (55mg de 
Resumo dos estudos realizados no Brasil para avaliar o impacto da fortificação de alimentos com ferro sobre a ocorrência de anemia.

\begin{tabular}{|c|c|c|c|c|c|c|c|}
\hline Referência & $\begin{array}{c}\text { Ano de } \\
\text { publicação }\end{array}$ & $\begin{array}{l}\text { Local de } \\
\text { estudo }\end{array}$ & $\mathbf{N}$ & $\begin{array}{l}\text { Tipo de } \\
\text { estudo }\end{array}$ & $\begin{array}{l}\text { Duração } \\
\text { (meses) }\end{array}$ & Resultado & $\begin{array}{l}\text { Alimento } \\
\text { fortificado }\end{array}$ \\
\hline $\begin{array}{l}\text { Nogueira } \\
\text { et al. } 31\end{array}$ & 1992 & Piauí & $\begin{array}{l}16 \text { crianças; } \\
2 \text { a } 4 \text { anos } \\
\text { incompletos; } \\
1 \text { creche }\end{array}$ & $\begin{array}{l}\text { Antes e } \\
\text { depois }\end{array}$ & 3 & $\begin{array}{l}\text { Concentração média de hemoglobina aumentou } \\
\text { significativamente ao final de três meses }\end{array}$ & Biscoitos \\
\hline $\begin{array}{l}\text { Torres } \\
\text { et al. } 32\end{array}$ & 1995 & São Paulo & $\begin{array}{l}335 \text { crianças; } \\
\text { menores de } 2 \\
\text { anos; } 107 \text { de } \\
\text { creches públicas } \\
\text { e } 228 \text { de uma } \\
\text { UBS }\end{array}$ & $\begin{array}{l}\text { Antes e } \\
\text { depois }\end{array}$ & 6 & $\begin{array}{l}\text { A prevalência de anemia entre as crianças das } \\
\text { creches passou de } 66,4 \% \text { para } 20,6 \% \text { e nas UBS } \\
\text { de } 72,8 \% \text { para } 18 \% \text {. A média de hemoglobina } \\
\text { aumentou } 1,3 \text { e 1,1 g/dL nas creches e UBS, } \\
\text { respectivamente }\end{array}$ & Leite em pó \\
\hline $\begin{array}{l}\text { Torres } \\
\text { et al. } 33\end{array}$ & 1996 & Angatuba & $\begin{array}{l}269 \text { crianças; } \\
6 \text { a } 42 \text { meses; } \\
\text { beneficiários de } \\
\text { programa de } \\
\text { suplementação } \\
\text { alimentar }\end{array}$ & $\begin{array}{l}\text { Antes e } \\
\text { depois }\end{array}$ & 12 & $\begin{array}{l}\text { Prevalência de anemia passou de } 62,3 \% \text { a } 41,8 \% \text {, } \\
\text { com redução maior nas crianças com menos de } \\
23 \text { meses }\end{array}$ & Leite \\
\hline $\begin{array}{l}\text { Torres } \\
\text { et al. } 34\end{array}$ & 1996 & Ibiúna & $\begin{array}{l}238 \text { crianças; } 6 \text { a } \\
18 \text { meses, uma } \\
\text { UBS }\end{array}$ & $\begin{array}{l}\text { Antes e } \\
\text { depois }\end{array}$ & 6 & $\begin{array}{l}\text { A prevalência de anemia caiu de } 72,6 \% \text { no início } \\
\text { do estudo para } 38,9 \% \text { e } 18,5 \% \text { aos } 3 \text { e } 6 \text { meses }\end{array}$ & Leite em pó \\
\hline $\begin{array}{l}\text { Fisberg } \\
\text { et al. } 35\end{array}$ & 1996 & São Paulo & $\begin{array}{l}125 \text { crianças; } \\
2 \text { a } 6 \text { anos; } 2 \\
\text { creches públicas }\end{array}$ & $\begin{array}{l}\text { Antes e } \\
\text { depois }\end{array}$ & 6 & $\begin{array}{l}\text { A média de hemoglobina aumentou } \\
\text { significativamente ao final do período de } \\
\text { intervenção. O número de crianças com níveis de } \\
\text { ferritina sérica inferior a } 10 \mathrm{mcg} / \mathrm{dL} \text { passou de } 23 \\
\text { para } 6\end{array}$ & $\begin{array}{l}\text { Fórmula } \\
\text { láctea }\end{array}$ \\
\hline $\begin{array}{l}\text { Vitolo } \\
\text { et al. } 36\end{array}$ & 1998 & São Paulo & $\begin{array}{l}54 \text { crianças; } 1 \text { a } \\
4 \text { anos; } 2 \text { classes } \\
\text { de uma creche } \\
\text { pública }\end{array}$ & $\begin{array}{l}\text { Antes e } \\
\text { depois }\end{array}$ & 2 & $\begin{array}{l}\text { Os valores médios de hemoglobina ao final da } \\
\text { intervenção foram significativamente maiores } \\
\text { para as crianças da classe I (alimentadas pelas } \\
\text { cuidadoras). No grupo de crianças da classe II } \\
\text { (alimentavam-se sozinhas) não houve diferença } \\
\text { significativa nas médias antes e depois da } \\
\text { intervenção. Quando foram consideradas todas as } \\
\text { crianças, conjuntamente, no grupo daquelas com } \\
\text { níveis de hemoglobina menores que } 11 \mathrm{~g} / \mathrm{dL} \text {, no } \\
\text { início do estudo, houve incremento significante na } \\
\text { média ao final do estudo }\end{array}$ & $\begin{array}{c}\text { Mingau de } \\
\text { cereal }\end{array}$ \\
\hline $\begin{array}{l}\text { Giorgini } \\
\text { et al. } 37\end{array}$ & 2001 & São Paulo & $\begin{array}{l}89 \text { crianças; } 12 \text { a } \\
72 \text { meses; baixa } \\
\text { renda; } 2 \text { creches } \\
\text { públicas }\end{array}$ & $\begin{array}{l}\text { Antes e } \\
\text { depois }\end{array}$ & 6 & $\begin{array}{l}\text { Prevalência de anemia diminuiu de } 62 \% \text { para } 22 \% \text {. } \\
\text { Aumento na média de hemoglobina de } 1,1 \mathrm{~g} / \mathrm{dL} \text {. } \\
\text { Média de ferritina plasmátca aumentou de } 11,3 \\
\text { para } 20,2 \mathrm{mcg} / \mathrm{L}\end{array}$ & $\begin{array}{c}\text { Massas e } \\
\text { doces }\end{array}$ \\
\hline $\begin{array}{l}\text { De Paula } \\
\text { et al. } 38\end{array}$ & 2001 & São Paulo & $\begin{array}{l}93 \text { crianças ; } 10 \\
\text { a } 48 \text { meses; } 1 \\
\text { creche pública }\end{array}$ & $\begin{array}{l}\text { Antes e } \\
\text { depois }\end{array}$ & 6 & $\begin{array}{l}\text { Aumento médio da hemoglobina de } 0,4 \mathrm{~g} / \mathrm{dL} \\
\text { ( } p<0,001 \text { ). Considerando somente as crianças } \\
\text { anêmicas (32/93) o incremento médio de } \\
\text { hemoglobina foi de } 1,4 \mathrm{~g} / \mathrm{dL}\end{array}$ & Açúcar \\
\hline
\end{tabular}

(continua) 


\begin{tabular}{|c|c|c|c|c|c|c|c|}
\hline Referência & $\begin{array}{c}\text { Ano de } \\
\text { publicação }\end{array}$ & $\begin{array}{l}\text { Local de } \\
\text { estudo }\end{array}$ & $\mathbf{N}$ & $\begin{array}{l}\text { Tipo de } \\
\text { estudo }\end{array}$ & $\begin{array}{l}\text { Duração } \\
\text { (meses) }\end{array}$ & Resultado & $\begin{array}{l}\text { Alimento } \\
\text { fortificado }\end{array}$ \\
\hline Tuma et al. 39 & 2003 & Manaus & $\begin{array}{l}80 \text { pré-escolares; } \\
2 \text { a } 6 \text { anos; } 1 \\
\text { creche pública; } 4 \\
\text { grupos }\end{array}$ & Randomizado & 4 & $\begin{array}{l}\text { A prevalência de anemia no grupo total de } \\
\text { crianças passou de } 22,7 \% \text {, no início do estudo, } \\
\text { para } 8 \% \text { no final. A média de hemoglobina ao final } \\
\text { do estudo foi significativamente maior quando } \\
\text { analisadas todas as crianças. No entanto, não } \\
\text { houve diferença na média final e no ganho de } \\
\text { hemoglobina entre os grupos }\end{array}$ & $\begin{array}{c}\text { Farinha de } \\
\text { mandioca } \\
\text { com } \\
\text { diferentes } \\
\text { concentrações } \\
\text { de ferro }\end{array}$ \\
\hline $\begin{array}{l}\text { Miglioranza } \\
\text { et al. } 40\end{array}$ & 2003 & Londrina & $\begin{array}{l}468 \text { crianças; } 7 \text { a } \\
14 \text { anos; centros } \\
\text { de educação } \\
\text { pública }\end{array}$ & $\begin{array}{l}\text { Antes e } \\
\text { depois }\end{array}$ & 12 & $\begin{array}{l}\text { A prevalência de anemia decresceu } \\
\text { significativamente de } 41,9 \% \text { no início do estudo, } \\
\text { para } 26,4 \% \text { aos seis meses e para } 9,6 \% \text { após um } \\
\text { ano. Aumento significativo dos níveis médios } \\
\text { de hemoglobina foi observado em todos os } \\
\text { acompanhamentos }\end{array}$ & Bebida láctea \\
\hline
\end{tabular}

ferro eletrolítico/100g de cereal) e de uma fórmula fortificada (12mg de sulfato ferroso/100g de pó) na prevenção da anemia em crianças com 4 a 15 meses de idade, atendidas em um ambulatório público. O delineamento utilizado foi o experimental controlado, aleatorizado, duplo cego. Inicialmente, 515 crianças foram distribuídas em dois grupos quanto ao tempo de aleitamento materno exclusivo (maior ou menor que quatro meses). Aquelas cujo aleitamento exclusivo foi interrompido antes dos quatro meses de idade foram aleatorizadas em três grupos. O grupo 1 recebeu cereal fortificado + fórmula não-fortificada; o grupo 2, cereal e fórmula não-fortificada e o grupo 3, cereal não-fortificado e fórmula fortificada. As crianças que estavam sendo amamentadas ao seio exclusivamente foram aleatorizadas em dois grupos, sendo que um grupo recebeu cereal fortificado e outro cereal não-fortificado. À medida que eram desmamadas, fórmulas não- fortificadas eram adicionadas à dieta. Dietistas faziam visitas domiciliares semanais para orientações e registro do consumo das fórmulas e do cereal. As perdas de acompanhamento chegaram a 20,6\%. Menos de $20 \%$ das crianças ingeriram menos de $24 \mathrm{~g}$ do cereal/dia. Inicialmente e aos 8, 12 e 15 meses de idade foram obtidas amostras de sangue capilar. As crianças do grupo 1 apresentaram valores significativamente mais altos de hemoglobina nos três acompanhamentos, quando comparadas ao grupo 2, que não recebeu fortificação (118 $\pm 1,0 \mathrm{x}$ $114 \pm 1,5 \mathrm{~g} / \mathrm{L} ; 124 \pm 1,0$ x $120 \pm 1,0 \mathrm{~g} / \mathrm{L} ; \mathrm{e} 126 \pm 1,1$ x $121 \pm 1,6 \mathrm{~g} / \mathrm{L})$. Não houve diferença significativa na concentração de hemoglobina entre o grupo que recebeu cereal fortificado e o que recebeu fórmula fortificada. Entre as crianças amamentadas, a concentração de hemoglobina foi mais alta aos 12 e 15 meses no grupo que recebeu leite materno mais cereal fortificado. A concentração basal de hemoglobina dos participantes e o poder do estudo para as análises entre os grupos não foram relatados.

Walter et al. 24 também estudaram o impacto da ingestão de 3 unidades (30g) de biscoitos fortificados com $6 \%$ de concentrado de hemoglobina bovina (aporte de $1 \mathrm{mg}$ de ferro biodisponível por dia), no status de ferro de 933 escolares de 10 a 16 anos em duas províncias do Chile, similares em termos de clima, agricultura e condição sócioeconômica. Na província controle, os escolares recebiam biscoitos similares, porém sem adição de ferro. Em cada província, foram selecionadas quatro escolas, duas urbanas e duas rurais, sendo incluídas no estudo somente as crianças que participavam do programa de alimentação destas escolas há mais de dois anos. Amostras de sangue venoso foram coletadas no início do estudo para análise de hemoglobina, hematócrito, eritrócitos e ferritina. A aceitabilidade dos biscoitos fortificados foi avaliada em estudo piloto realizado anteriormente, sendo de $97 \%$. Apesar da baixa prevalência de anemia em ambos os grupos, houve diferença significativa na concentração de hemoglobina e ferritina sérica, favorecendo as crianças que consumiram biscoitos fortificados após três anos de intervenção.

Na Guatemala, Viteri et al. 25 conduziram um estudo controlado, randomizado, duplo cego, envolvendo quatro comunidades semi-rurais de baixa renda. Uma comunidade recebeu açúcar fortificado com vitamina A (comunidade contro- 
le) e três receberam açúcar fortificado com vitamina A e ferro (etileno diamino tetra acetato - EDTA de ferro e sódio, na quantidade de $1 \mathrm{~g} / \mathrm{kg}$ de açúcar). Todos os distribuidores de açúcar e varejistas foram contatados pela equipe de pesquisa e concordaram em adquirir e revender somente açúcar fortificado, o qual foi vendido aos mesmos com desconto. Os comerciantes registravam a quantidade de açúcar adquirida pelas famílias e as queixas em relação ao produto. Periodicamente, durante o estudo, amostras de açúcar foram obtidas nos domicílios para análise do conteúdo em ferro. A adesão ao consumo do açúcar fortificado foi estimada para cada domicílio e expressa como um percentual de amostras positivas para ferro. Esses testes indicaram que a comunidade controle não dispunha de açúcar fortificado. Nas comunidades que receberam açúcar com adição de ferro, o percentual de domicílios com testes positivos variou de $72 \%$ a $93 \%$. As queixas com relação ao produto foram poucas (oito em 32 meses de estudo). Em média, 87 crianças de um a oito anos de idade foram avaliadas em cada comunidade. O percentual de perdas por comunidade estudada variou de $29 \%$ a $43 \%$. Essas perdas não diferiram daqueles que continuaram no estudo quanto aos indicadores hematológicos, status de ferro e condições nutricionais. Ao final de 32 meses de acompanhamento, para ambos os sexos, na comunidade controle não houve aumento significativo, em comparação à análise basal, nas medidas de hemoglobina, eritrócitos, ferritina e estoques de ferro, mas sim na capacidade de saturação da transferrina em amostras de sangue venoso. Nas três comunidades que sofreram intervenção, houve manutenção ou aumento significativo em todos esses indicadores do status de ferro.

Layrisse et al. 26, na Venezuela, avaliaram a resposta à fortificação universal de farinhas de milho pré-cozido e de trigo, com 50 e $20 \mathrm{mg}$ de ferro (fumarato ferroso) por quilograma de farinha, respectivamente, implantada em 1993. As prevalências de deficiência de ferro (determinada por concentração de ferritina sérica) e de anemia (determinada pela concentração de hemoglobina e volume dos eritrócitos), medidas em amostras de sangue venoso, foram avaliadas em 317 adolescentes escolares de 7, 11 e 15 anos de idade e de baixo nível sócio-econômico, moradores em Caracas. Os resultados foram comparados aos de dois inquéritos populacionais de representatividade nacional, conduzidos em 1989-1990 e 1992. A análise dos dados mostrou que a prevalência de deficiência de ferro que era de $14 \%$ em 19891990 passou a 36,6\%, em 1992, e 15,8\%, em 1994. A prevalência de anemia foi de 3,6\%, 19\% e 9,3\% em 1989-1990, 1992 e 1994, respectivamente. O efeito de fatores externos ao programa não foi considerado na análise.

Em outro estudo conduzido no Chile, Walter et al. 27, utilizando como delineamento um ensaio clínico aleatorizado duplo cego, testaram o efeito de fórmulas lácteas com alta e baixa concentração de ferro $(12,7 \mathrm{mg} / \mathrm{L}$ ou $2,3 \mathrm{mg} / \mathrm{L}$ de sulfato ferroso, respectivamente) sobre a anemia em crianças saudáveis, com seis meses de idade, de quatro clínicas comunitárias. Consentimento para participação no estudo foi obtido de 94,3\% dos pais, resultando em uma amostra de 1.179 crianças. Cinqüenta e nove crianças foram excluídas por estarem com deficiência de ferro, anemia ou altos níveis de hemoglobina. As 1.120 restantes foram aleatorizadas para receber as diferentes fórmulas. As perdas, igualmente distribuídas entre os grupos, totalizaram 25,4\% ( $\mathrm{n}=$ 285). Assim, 835 crianças completaram o protocolo do estudo, 405 recebendo fórmula com baixa concentração de ferro e 430, fórmula com alta concentração desse mineral. Visitas domiciliares semanais eram realizadas para checar a adesão ao consumo das fórmulas, que foi similar nos dois grupos. Aos 12 meses de idade, uma amostra de sangue venoso foi obtida para análise do status de ferro. As duas fórmulas foram efetivas na prevenção da anemia, não havendo diferença na prevalência (respectivamente $2,8 \%$ no grupo com $12,7 \mathrm{mg} / \mathrm{L}$ e $3,8 \%$ no grupo com $2,3 \mathrm{mg} / \mathrm{L}$ ).

Utilizando balas fortificadas com ferro elementar, Sari et al. 28 conduziram um ensaio clínico, duplo cego, placebo controlado, com alocação aleatória de 132 crianças de baixa e média renda, com quatro a seis anos de idade, de quatro escolas infantis em Jakarta, Indonésia. As balas fortificadas que proporcionavam às crianças um total de 30mg de ferro/semana ou as balas placebo foram distribuídas três vezes por semana. Os autores não mencionaram como a adesão ao consumo das balas foi controlada, citando apenas a opinião das mães e crianças sobre as mesmas. O percentual de perdas foi de 11\%. Após 12 semanas, a concentração média de hemoglobina em sangue venoso no grupo que recebeu balas fortificadas aumentou $10,2 \mathrm{~g} / \mathrm{L}$, enquanto que no grupo controle aumentou $4,0 \mathrm{~g} / \mathrm{L}$ $(\mathrm{p}<0,001)$. A prevalência de anemia decresceu de $50,9 \%$ para $8,8 \%$ e de $43,3 \%$ para $26 \%$ nos grupos intervenção e controle, respectivamente. O mesmo aconteceu com a concentração de ferritina sérica que foi $71 \%$ e $28 \%$ mais alta do que no baseline, nos grupos intervenção e controle, respectivamente.

Nestel et al. 29 avaliaram a efetividade do uso de farinhas de trigo fortificadas com ferro eletrolítico ou reduzido na concentração de $66 \mathrm{mg} / \mathrm{kg}$, na diminuição da prevalência de ane- 
mia por meio de um ensaio clínico controlado, duplo cego, conduzido por 24 meses. O estudo foi realizado para que o governo avaliasse a decisão de estabelecer a fortificação compulsória das farinhas no país. O efeito da intervenção foi medido em pré-escolares de 9 a 71 meses e em escolares de 6 a 11 anos. Essa população foi selecionada em seis Estados do Sri Lanka. Foram reunidos 3.228 participantes, sendo $23 \%$ préescolares e $27 \%$ escolares. Destes, $62 \%$ e $45 \%$, respectivamente, foram perdidos no acompanhamento, sendo a perda maior no grupo que recebeu farinha fortificada com ferro eletrolítico. Três grupos de estrutura e tamanho similares foram formados aleatoriamente para receber farinhas fortificadas com ferro eletrolítico, reduzido ou não-fortificadas. A distribuição da farinha enriquecida ou não- enriquecida com ferro foi monitorada quinzenalmente, nos Estados, durante os dois anos de estudo. O consumo médio de farinhas foi de $120 \mathrm{~g}$ e $150 \mathrm{~g} /$ dia para pré-escolares e escolares, respectivamente. Nenhum dos dois tipos de farinha de trigo fortificada foi capaz de promover aumento na concentração média de hemoglobina, medida em sangue capilar, em pré-escolares e escolares, após um e dois anos de consumo.

Em estudo conduzido na África do Sul, van Stuijvenberg et al. 30 avaliaram a eficácia do consumo de pão preto enriquecido com dois tipos de compostos de ferro sobre a anemia, em 160 escolares de 6 a 11 anos de idade, com níveis séricos de ferritina menores que $20 \mathrm{mcg} / \mathrm{L}$. As crianças foram distribuídas aleatoriamente em três grupos. Um primeiro grupo recebeu pão não-fortificado; o segundo, pão fortificado com ferro eletrolítico; e o terceiro, pão fortificado com bisglicinato ferroso $(35 \mathrm{mg} / \mathrm{kg}$ de farinha). Cada criança recebeu quatro fatias $(120 \mathrm{~g})$ de pão por dia, sob supervisão dos professores. Foi coletado sangue venoso no início do estudo e ao final de sete meses e meio. As crianças receberam medicação para vermes (mebendazol) antes do estudo e seis meses mais tarde. Sete crianças $(4,4 \%)$ não completaram o estudo: duas controles e cinco do grupo que recebeu fortificação com ferro bisglicinato. Após sete meses, apenas o grupo que consumiu o pão com adição de bisglicinato ferroso apresentou um incremento significativo da medida de hemoglobina, ferro sérico e saturação da transferrina.

\section{Estudos no Brasil}

No Brasil, poucos estudos avaliando a eficácia ou a efetividade de fortificação de alimentos com ferro têm sido publicados 41 (Tabela 2). Nogueira et al. 31 avaliaram o efeito da introdução de biscoitos fortificados com hemoglobina bovina no estado de nutrição de ferro em pré-escolares de 2 a 4 anos incompletos, atendidos em uma creche no Estado do Piauí. A amostra inicial de 30 crianças ficou reduzida a 16 no final do estudo. Inicialmente, foram realizados exames de fezes e afastada a presença de parasitas intestinais relacionados à etiologia da anemia. As crianças passaram a ingerir, diariamente, além da dieta habitual, cinco biscoitos fortificados que totalizavam aproximadamente $4 \mathrm{mg}$ de ferro. Nos finais de semana, as mães ficavam responsáveis pela oferta dos biscoitos às crianças. Os autores relatam adesão de $100 \%$ ao consumo de biscoitos, mas não explicitam como essa foi avaliada. Três meses após, a concentração média de hemoglobina em sangue venoso das dezesseis crianças avaliadas antes e após a intervenção, passou de 9,4 \pm $2,6 \mathrm{~g} / \mathrm{dL}$ para $13,2 \pm 0,2 \mathrm{~g} / \mathrm{dL}$.

$\mathrm{O}$ efeito do uso do leite em pó integral fortificado com ferro e vitamina $\mathrm{C}$ sobre os níveis de hemoglobina de crianças de 6 a 23 meses foi avaliado por Torres et al. 32 . Os autores não mencionam a forma do ferro utilizado. Foram incluídas 107 crianças provenientes de 13 creches municipais e 228 usuárias de uma Unidade Básica de Saúde (UBS) do Estado de São Paulo. Leite fortificado com $9 \mathrm{mg}$ de ferro e $65 \mathrm{mg}$ de vitamina C por $100 \mathrm{~g}$ de pó, substituiu o leite em pó integral, não-fortificado, que era oferecido anteriormente nas creches. Na UBS o leite fortificado era distribuído às mães, sendo as mesmas orientadas sobre o preparo das mamadeiras. Nenhuma outra modificação ou orientação foi feita com relação aos cardápios das crianças estudadas. Avaliações dos níveis de hemoglobina foram realizadas três e seis meses após o início da intervenção, sendo obtidas por meio de coleta de sangue venoso. As médias de hemoglobina nas creches e na UBS aumentaram significativamente em relação ao início do estudo. A estratificação da amostra por status basal de anemia evidenciou que as médias de hemoglobina somente não foram significativamente maiores no final do estudo no grupo de crianças inicialmente não anêmicas, provenientes das creches.

Torres et al. 33 estudaram o impacto do uso de leite fortificado com ferro (aminoácido quelato) no combate à anemia em crianças menores de quatro anos. Foram selecionadas 269 crianças de 6 a 42 meses de idade, matriculadas no Programa de Suplementação Alimentar da Secretaria de Saúde e da Prefeitura Municipal de Angatuba, interior de São Paulo. As crianças receberam, diariamente, por 12 meses, um litro de leite fortificado com $3 \mathrm{mg}$ de ferro. Por meio de avaliações trimestrais, foram obtidas informações sobre a ingestão média diária, aceitação, composição fa- 
miliar e distribuição intrafamiliar do suplemento. Após seis meses de intervenção, a prevalência de anemia, avaliada utilizando-se os níveis de hemoglobina obtidos por punção digital, passou de $62,3 \%$ para $41,8 \%$ e, ao final de 12 meses, para $26,4 \%$. As maiores reduções ocorreram entre os 12 e 23 meses de idade. As médias de hemoglobina apresentaram incrementos significativos no grupo de crianças com menos de 36 meses de idade e nas com anemia grave (hemoglobina < 9,5g/dL) e moderada (hemoglobina entre 9,5 e $10,9 \mathrm{~g} / \mathrm{dL}$ ). A melhora na evolução da hemoglobina foi maior naqueles que ingeriam quantidades de leite superiores a 750ml/dia, provenientes de famílias que não dividiam o leite do beneficiário do programa com outros membros.

Em outro estudo, conduzido em município da Grande São Paulo (Ibiúna), Torres et al. 34 acompanharam crianças com idade compreendida entre 6 e 18 meses, matriculadas em programa de suplementação alimentar e captadas em uma UBS. As crianças, após avaliação clínica e hematológica inicial (sangue capilar), passaram a receber leite em pó fortificado com $9 \mathrm{mg}$ de ferro elementar e $65 \mathrm{mg}$ de vitamina $C$ por cada $100 \mathrm{~g}$ de pó, por seis meses. As mães recebiam o leite em quantidades mensais pré-determinadas e eram orientadas quanto ao preparo. Cada criança deveria receber duas a três mamadeiras com $250 \mathrm{ml}$ de leite por dia, sendo que cada uma fornecia $3 \mathrm{mg}$ de ferro. Das 241 crianças avaliadas inicialmente, 228 foram reavaliadas aos três meses e 238 aos seis meses de intervenção. Nos acompanhamentos trimestrais foram obtidas informações sobre o preparo do leite, quantidade ingerida por dia e a distribuição intrafamiliar do produto. Após três meses de ingestão de leite fortificado, a prevalência de anemia diminuiu de $72,6 \%$ para $38,9 \%$ e, aos seis meses, para $18,5 \%$ $(\mathrm{p}<0,001)$.

Fisberg et al. 35 estudaram 125 crianças de dois a seis anos, de duas creches do Município de São Paulo. Diariamente, no horário do lanche, eram oferecidos, em substituição ao leite tradicionalmente utilizado, $350 \mathrm{ml}$ de um suplemento lácteo industrializado, que continha $1,4 \mathrm{mg}$ de ferro/100ml. A ingestão do mesmo era anotada pelas cuidadoras e supervisionada pela equipe do projeto. Oitenta e nove por cento das crianças aceitaram sempre o suplemento, sem recusas, sendo que a média de ingestão foi de $347 \mathrm{ml} /$ dia. O estudo teve $18 \%$ de perdas de acompanhamento. Todas as crianças foram avaliadas quanto à hemoglobina e ferritina sérica (sangue venoso) no início do estudo e aos 90 e 180 dias após o ingresso. A média dos níveis de hemoglobina aumentou significativamente do início até o término da intervenção. O número de crianças com ferritina sérica abaixo de 10mcg/dL passou de 23 para seis casos, ao final de 180 dias.

Vítolo et al. 36 realizaram um estudo com o objetivo de avaliar o efeito de um mingau de farinhas de cereais adicionado de ortofosfato de ferro $(12 \mathrm{mg} / 100 \mathrm{~g})$ sobre os níveis de hemoglobina de pré-escolares. Foram estudadas 54 crianças de um a quatro anos de idade, de duas classes de uma creche do Município de São Paulo. As 24 crianças da classe I tinham em média 2,2 anos e se alimentavam com ajuda das auxiliares e professoras, enquanto as 30 da classe II tinham em média 3,6 anos e se alimentavam sozinhas. $\mathrm{O}$ produto (15g para $200 \mathrm{ml}$ de leite) foi oferecido duas vezes por dia, substituindo o café da manhã e o lanche da tarde, durante oito semanas. A quantidade consumida pelas crianças foi avaliada diariamente por meio de pesagem direta das sobras do mingau. A aceitação melhorou após a segunda semana do estudo. Os valores médios de hemoglobina, medidos antes e no final da intervenção, foram significativamente maiores para as crianças da classe I, com média inicial de 9,9 $\pm 2,0 \mathrm{~g} / \mathrm{dL}$ e final de $11,4 \pm 1,0 \mathrm{~g} / \mathrm{dL}(\mathrm{p}<0,01)$. No grupo de crianças da classe II não houve diferença significativa nas médias antes e depois da intervenção. Quando foram consideradas todas as crianças, conjuntamente, os autores observaram que, no grupo daquelas com níveis de hemoglobina menores que $11 \mathrm{~g} / \mathrm{dL}$ no início do estudo houve incremento médio significante na média ao final do estudo. Houve perda de $22 \%$ da amostra na análise final dos níveis de hemoglobina por recusa em fazer o exame ou não comparecimento à creche (6 crianças na classe I e 6 na classe II).

Giorgini et al. 37 conduziram um estudo com 92 crianças com idade entre 12 e 72 meses, de baixo nível sócio-econômico, atendidas em creches da cidade de São Paulo. Além de sua dieta habitual, as crianças receberam por seis meses, cinco vezes por semana, duas porções diárias de uma massinha doce de $25 \mathrm{~g}$, fortificadas com $2 \mathrm{mg}$ de ferro (quelato), totalizando $4 \mathrm{mg}$ de ferro/dia. A adesão ao produto não está descrita no artigo. Três crianças não completaram o estudo (3,3\%). Ao final de seis meses de intervenção, a prevalência de anemia (hemoglobina $<11 \mathrm{~g} / \mathrm{dL}$ ), obtida por meio da análise de sangue venoso, passou de $28 \%$ para $9 \%$. Os níveis médios de hemoglobina e ferritina aumentaram significativamente depois da intervenção, sendo este aumento ainda maior em crianças inicialmente anêmicas.

Estudo de Cardoso-de-Paula et al. 38 avaliou o efeito do consumo de açúcar fortificado com ferro (triglicinato quelato) sobre anemia em 93 crianças com idade entre 10 e 48 meses, de uma creche, em São Paulo. As crianças foram distribuídas em dois grupos. O grupo $1(n=42)$ recebeu 
açúcar fortificado com $10 \mathrm{mg}$ de ferro/ $\mathrm{kg}$ e o grupo $2(\mathrm{n}=51)$ recebeu fortificação de $100 \mathrm{mg} / \mathrm{kg}$. Vinte gramas de ambos os tipos de açúcar foram ingeridos por dia, adicionados a suco de laranja, com boa aceitabilidade. Foi coletado sangue venoso para as análises de hemoglobina e ferritina. Não houve perdas de acompanhamento. Aumento médio de $0,4 \mathrm{~g} / \mathrm{dL}$ de hemoglobina foi observado nos dois grupos, após seis meses de consumo do açúcar. Analisando somente as crianças com anemia ( $\mathrm{Hb}$ inicial $<11 \mathrm{~g} / \mathrm{dL}$ ), os autores observaram um incremento médio da hemoglobina de 1,3 e 1,5g/dL nos grupos 1 e 2, respectivamente. A prevalência média de anemia decresceu $43,8 \%$ no grupo 1 e $66,7 \%$ no grupo 2 . Os níveis séricos de ferritina só foram diferentes nos dois momentos entre as crianças com baixos níveis iniciais. Nestas, houve acréscimo de 5,0 para 12,2mcg/L no grupo 1 e de 6,5 para $15,9 \mathrm{mcg} / \mathrm{L}$ no grupo 2 .

Tuma et al. ${ }^{39}$ realizaram um ensaio clínico randomizado com 80 pré-escolares, de dois a seis anos, matriculados em uma creche filantrópica de Manaus, distribuídos em quatro grupos. Três grupos receberam farinha de mandioca adicionada de ferro (quelato) durante 120 dias, no horário do almoço, na quantidade de 5, 10 e 15g, respectivamente. Essas quantidades de farinha proporcionavam a ingestão de 1, 2 e 3mg de ferro/dia. As crianças que compunham o quarto grupo receberam $5 \mathrm{~g}$ de farinha de mandioca sem adição de ferro, pelo mesmo período de tempo. A perda amostral foi de $6 \%$. A prevalência de anemia, determinada por meio da análise de sangue venoso no grupo total de crianças, passou de $22,7 \%$, no início do estudo, para $8 \%$ no final. No grupo 1, passou de $25 \%$ a $15 \%$; no grupo 2, de $21 \%$ para $0 \%$; no grupo 3 , de $32 \%$ para $16 \%$; e, no grupo 4 , de $12 \%$ para $0 \%$. Não houve diferença na média final e no ganho de hemoglobina entre os grupos estudados.

Em Londrina, Estado do Paraná, Miglioranza et al. 40 investigaram o efeito de uma bebida láctea fortificada com ferro (quelato $-12 \mathrm{mg} / 100 \mathrm{ml}$ ), nos valores de hemoglobina em crianças e adolescentes assistidos por nove centros públicos de educação. Quatrocentas e sessenta e oito crianças entre 7 e 14 anos foram avaliadas no início e aos 3, 6 e 12 meses do estudo. Cada criança ingeria $100 \mathrm{ml} /$ dia da bebida, que continha $12 \mathrm{mg}$ de ferro. A perda amostral chegou a $33 \%$ aos 12 meses de estudo. A prevalência de anemia, avaliada por meio de sangue capilar, decresceu significativamente de $41,9 \%$, no início do estudo, para $26,4 \%$ aos seis meses e 9,6\% após um ano. Aumento significativo dos níveis médios de hemoglobina foi observado em todos os acompanhamentos.

\section{Discussão}

De acordo com os critérios de Downs \& Black 19 para avaliação da qualidade metodológica dos estudos, a pontuação máxima alcançável seria de 31 pontos. O escore médio observado foi baixo: 11,3 pontos $(\mathrm{DP}=6,4$; mediana $=10,0)$ para os estudos internacionais, e 3,1 ( $D P=0,8$; mediana $=3,0$ ) para os nacionais. Apenas dois estudos alcançaram pontuação superior a vinte: o de van Stuijvenberg et al. 30 , com 22 pontos, e o de Nestel 29, com 21.

A maioria dos estudos apresentou limitações que podem comprometer sua validade interna. As limitações metodológicas mais importantes detectadas foram a não apresentação do poder do estudo; a utilização de mais de dois grupos de participantes com tamanhos pequenos; a não descrição ou o excessivo número de perdas e as potenciais implicações das mesmas sobre os resultados; a falta de identificação e controle de fatores de confusão; a ausência de informações basais sobre os índices hematimétricos estudados; e o fato de não levar em conta, na análise, o efeito de conglomerado utilizado na seleção amostral (unidade amostral agrupada, como escolas, salas de aula, ambulatórios etc. e unidade de análise individual). Tais limitações contra-indicam, inclusive, o cálculo de uma medida agregada de efeito, que poderia ser obtida por meio de uma meta-análise.

Dois fatos merecem destaque. Primeiro, os dois estudos que apresentaram os maiores escores metodológicos 29,30 encontraram resultados opostos. Segundo, somente um estudo 29 não mostrou impacto da fortificação de alimentos com ferro na ocorrência de anemia. Esses fatos apontam para a possibilidade de existência de viés de publicação, ao mesmo tempo em que indicam a necessidade de realização de outros estudos.

Embora a fortificação de alimentos com ferro venha sendo utilizada como uma estratégia para combater a deficiência de ferro em muitos países, a documentação existente sobre seu impacto na população é muito limitada ${ }^{42}$. Nesta revisão, identificou-se apenas um estudo que avaliou a efetividade de fortificação universal já implantada em um país 26 , mostrando um impacto positivo na prevalência de anemia. Nesse estudo, no entanto, os autores não tentaram descartar o efeito de outros fatores, externos ao programa de fortificação, que pudessem atuar sobre o resultado encontrado. Embora seja válida em termos locais, a capacidade de generalização dos achados para outros contextos sociais fica comprometida. Além disso, apenas escolares foram avaliados, o que limita a representatividade da 
amostra na avaliação de um programa de cunho universal.

A atual revisão deixa claro que há poucos dados que sustentem o efeito do aumento da ingestão de ferro a partir de alimentos fortificados (fortificação em massa ou direcionada) na melhoria do estado nutricional de ferro 13. Conseqüentemente, embora desejável, a eficácia e a efetividade da fortificação de alimentos com ferro sobre a prevalência de anemia, na população infantil, ainda está por ser demonstrada.

\section{Resumo}

Uma revisão sistemática da literatura foi conduzida com o objetivo de identificar estudos que avaliassem o efeito da fortificação de alimentos com ferro sobre a ocorrência de anemia em crianças. As bases de dados pesquisadas foram MEDLINE, LILACS e sites da Organização Mundial da Saúde e Organização Pan-Americana da Saúde, sem limite de tempo, incluindo estudos publicados em português, inglês ou espanhol, utilizando os seguintes unitermos e suas combinações: food fortification, iron, effectiveness, efficacy, anemia, flour, staple foods, interventions $e$ children. Dos 21 estudos revisados, apenas um não mostrou efeito positivo da intervenção, indicando a possibilidade de existência de viés de publicação. Os estudos apresentaram limitações metodológicas importantes. Os dois estudos com os melhores escores metodológicos mostraram resultados discordantes, evidenciando a necessidade de explorar essa hipótese em ensaios maiores e com maior rigor metodológico.

Alimentos Fortificados; Ferro; Anemia; Bem-Estar da Criança

\section{Colaboradores}

M. C. F. Assunção estruturou o conteúdo, analisou os estudos e redigiu a versão do manuscrito. I. S. Santos orientou a redação e fez a revisão do manuscrito. 


\section{Referências}

1. Baltussen R, Knai C, Sharan M. Iron fortification and iron supplementation are cost-effective interventions to reduce iron deficiency in four subregions of the world. J Nutr 2004; 134:2678-84.

2. World Health Organization/United Nations Children's Fund/United Nations University. Iron deficiency anaemia. Assessment, prevention and control. A guide for programme managers. Geneva: World Health Organization/United Nations Children's Fund/United Nations University; 2001.

3. Monteiro CA, Szarfarc SS, Mondini L. Tendência secular da anemia na infância na cidade de São Paulo. Rev Saúde Pública 2000; 36:62-72.

4. Fundo das Nações Unidas para a Infância. Crianças e adolescentes no Piauí. Saúde, educação e trabalho. Brasília: Fundo das Nações Unidas para a Infância; 1992.

5. Monteiro CA, Szarfarc SC. Estudos das condições de saúde das crianças no Município de São Paulo, SP (Brasil), 1984-1985. Rev Saúde Pública 1987; 21:255-60.

6. Instituto Nacional de Alimentação e Nutrição/Instituto Materno-Infantil de Pernambuco/Secretaria Estadual de Saúde de Pernambuco. 2a Pesquisa Estadual de Saúde e Nutrição: saúde, nutrição, alimentação e condições socioeconômicas no Estado de Pernambuco. Recife: Instituto Nacional de Alimentação e Nutrição, Ministério da Saúde; 1998.

7. Martins M, Santos LM, Lima A, Assis A, Amorim D. Perfil nutricional: desnutrição e anemia em crianças no Estado de Sergipe. In: 5o Congresso Nacional da Sociedade Brasileira de Alimentação e Nutrição. São Paulo: Sociedade Brasileira de Alimentação e Nutrição; 1999. p. 184.

8. Assis AMO, Barreto ML, Santos LMP, Sampaio LR, Magalhães LP, Prado MS, et al. Condições de vida, saúde e nutrição na infância em Salvador. Salvador: Escola de Nutrição/Instituto de Saúde Coletiva, Universidade Federal da Bahia; 2000.

9. Neuman NA, Tanaka OY, Szarfarc SC, Guimarães PRV, Victora CG. Prevalência e fatores de risco para anemia no Sul do Brasil. Rev Saúde Pública 2000; 34:56-63.

10. Osório MM, Lira PIC, Batista-Filho M, Ashworth A. Prevalence of anemia in children 6-59 months old in the state of Pernambuco, Brazil. Rev Panan Salud Pública 2001; 10:101-7.

11. Oliveira RS, Diniz AS, Benigna MJC, Miranda-Silva SM, Lola MM, Gonçalves MC, et al. Magnitude, distribuição espacial e tendência da anemia em pré-escolares da Paraíba. Rev Saúde Pública 2002; 36:26-32.

12. Lima ACVMS, Lira PIC, Romani SAM, Eickmann SH, Piscoya MD, Lima MC. Fatores determinantes dos níveis de hemoglobina em crianças aos 12 meses de vida na Zona da Mata Meridional de Pernambuco. Rev Bras Saúde Matern Infant 2004; 4:35-43.

13. International Nutritional Anemia Consultative Group. Efficacy and effectiveness of interventions to control iron deficiency and iron deficiency anemia. Washington DC: International Life Sciences Institute; 2004
14. Lynch SR. The impact of iron fortification on nutritional anaemia. Best Pract Res Clin Haematol 2005; 18:333-46.

15. Trowbridge F, Martorell R. Summary and recommendations. J Nutr 2002; 132:875S-9S.

16. Gleason GR, Sharmanov T. Anemia prevention and control in four Central Asian Republics and Kazakhstan. J Nutr 2002; 132:867S-70S.

17. Organización Panamericana de la Salud/Centers for Disease Control and Prevention. 1o Taller sobre monitoreo regulatorio en programas de fortificatión de harinas de trigo. http://www.paho.org (acessado em 08/Fev/2006).

18. Agência Nacional de Vigilância Sanitária. Resolução n. 344. Revoga a resolução - RDC n. 15, de 21 de fevereiro de 2000. Diário Oficial da União 2002; $18 \mathrm{dez}$.

19. Downs SH, Black N. The feasibility of creating a checklist for the assessment of the methodological quality both of randomised and non-randomised studies of health care interventions. J Epidemiol Community Health 1998; 52:377-84.

20. Hertrampf E, Olivares M, Walter T, Pizarro F, Heresi $\mathrm{G}$, Llaguno S, et al. Anemia ferropriva em el lactante: erradicación com leche fortificada com hierro. Rev Med Chile 1990; 118:1330-7.

21. Hertrampf E, Olivares M, Pizarro F, Walter T, Cayaz zo M, Heresi G, et al. Haemoglobin fortified cereal: a source of available iron to breast-fed infants. Eur J Clin Nutr 1990; 44:793-8.

22. Olivares M, Hertrampf E, Pizarro F, Walter T, Cayazzo M, Llaguno S, et al. Hemoglobin fortified biscuits: bioavailability and its effect on iron nutriture in school children. Arch Latinoam Nutr 1990; 40:209-20.

23. Walter T, Dallman PR, Pizarro F, Velozo L, Peña G Bartholmey SJ, et al. Effectiveness of iron-fortified infant cereal in prevention of iron deficiency anemia. Pediatrics 1993; 91:976-82.

24. Walter T, Hertrampf E, Pizarro F, Olivares M, Llaguno S, Letelier A, et al. Effect of bovine-hemoglobinfortified cookies on iron status of schoolchildren: a nationwide program in Chile. Am J Clin Nutr 1993; 57:190-4.

25. Viteri FE, Alvarez E, Batres R, Torún B, Pineda O, Mejía LA, et al. Fortification of sugar with iron sodium ethylenediaminotetraacetate (FeNaEDTA) improves iron status in semirural Guatemalan populations. Am J Clin Nutr 1995; 61:1153-63.

26. Layrisse M, Chaves JF, Mendez-Castellano H, Bosch V, Tropper E, Bastardo B, et al. Early response to the effect of iron fortification in the Venezuelan population. Am J Clin Nutr 1996; 64:903-7.

27. Walter T, Pino P, Pizarro F, Lozoff B. Prevention of iron-deficiency anemia: comparison of high-andlow-iron formulas in term infants after six months of life. J Pediatr 1998; 132:635-40

28. Sari M, Bloem MW, de Pee S, Schultink WJ, Sastroamidjojo S. Effect of iron-fortified candies on the iron status of children aged 4-6 y in East Jakarta, Indonesia. Am J Clin Nutr 2001; 73:1034-9. 
29. Nestel P, Nalubola R, Sivakaneshan R, Wickramasinghe AR, Atukorala S, Wickramannayake T, et al. The use of iron-fortified wheat flour to reduce anemia among the state population in Sri Lanka. Int J Vitam Nutr Res 2004; 74:35-51.

30. van Stuijvenberg ME, Smuts CM, Wolmarans P, Lombard CJ, Dhansay MA. The efficacy of ferrous bisglycinate and electrolytic iron as fortificants in bread in iron-deficient school children. Br J Nutr 2006; 95:532-8.

31. Nogueira NN, Colli C, Cozzolino SMF. Controle da anemia ferropriva em pré-escolares por meio da fortificação de alimento com concentrado de hemoglobina bovina (estudo preliminar). Cad Saúde Pública 1992; 8:459-65.

32. Torres MAA, Sato K, Lobo NF, Queiroz SS. Efeito do uso de leite fortificado com ferro e vitamina $\mathrm{C}$ sobre os níveis de hemoglobina e condição nutricional de crianças menores de 2 anos. Rev Saúde Pública 1995; 29:301-7.

33. Torres MAA, Lobo NF, Sato K, Queiroz SS. Fortificação do leite fluido na prevenção e tratamento da anemia carencial ferropriva em crianças menores de 4 anos. Rev Saúde Pública 1996; 30:350-7.

34. Torres MAA, Sato K, Juliano Y, Queiroz SS. O leite em pó fortificado com ferro e vitamina C como medida de intervenção no combate à anemia carencial ferropriva em crianças atendidas em Unidade Básica de Saúde. Arch Latinoam Nutr 1996; 46:113-7.

35. Fisberg M, Braga JA, Tadei JAA, Ferreira AMA, Kliamca PE, Schmidt BJ. Utilização de suplemento alimentar enriquecido com ferro na prevenção de anemia em pré-escolares. Revista de Pediatria Moderna 1996; 32:753-8.
36. Vítolo MR, Aguirre ANC, Kondo MR, Giuliano Y, Ferreira N, Lopez FA. Impacto do uso de cereal adicionado de ferro sobre níveis de hemoglobina e a antropometria em pré-escolares. Rev Nutr 1998; 11:163-71.

37. Giorgini E, Fisberg M, de Paula RA, Ferreira AM, Valle J, Braga JA. The use of sweet rolls fortified with iron bis-glycinate chelate in the prevention of iron deficiency anemia in preschool children. Arch Latinoam Nutr 2001; 51:48-53.

38. de Paula R, Fisberg M. The use of sugar fortified with iron tris-glycinate chelate in the prevention of iron deficiency anemia in preschool children. Arch Latinoam Nutr 2001; 51:54-9.

39. Tuma RB, Yuyama LKO, Aguiar JPL, Marques HO. Impacto da farinha de mandioca fortificada com ferro aminoácido quelato no nível de hemoglobina de pré-escolares. Rev Nutr 2003; 16:29-39.

40. Miglioranza LHS, Matsuo T, Caballero-Córdoba GM, Dichi JB, Cyrino ES, Oliveira IBN, et al. Effect of long-term fortification of whey drink with ferrous bisglycinate on anemia prevalence in children and adolescents from deprived areas in Londrina, Paraná, Brazil. Nutrition 2003; 19:419-21.

41. Beinner MA, Lamounier JA. Recent experience with fortification of foods and beverages with iron for the control $\mathrm{f}$ iron-deficiency anemia in Brazilian children. Food Nutr Bull 2003; 24:268-74.

42. Uauy R, Hertrampf E, Reddy M. Iron fortification of foods: overcoming technical and practical barriers. J Nutr 2002; 132:849S-52S.

Recebido em 04/Jul/2006

Aprovado em 04/Set/2006 